\title{
Apparent solar radius variations
}

\section{The influence of magnetic network and plage}

\author{
J. H. M. J. Bruls ${ }^{1}$ and S. K. Solanki ${ }^{2}$ \\ 1 Kiepenheuer-Institut für Sonnenphysik, Schöneckstr. 6, 79104 Freiburg, Germany \\ e-mail: bruls@kis.uni-freiburg.de \\ 2 Max-Planck Institut für Aeronomie, Max-Planck-Str. 2, Katlenburg-Lindau, Germany \\ e-mail: solanki@linmpi.mpg.de
}

Received 29 August 2003 / Accepted 22 July 2004

\begin{abstract}
Solar radius measurements, a by-product of the magnetograms recorded several times daily at Mt. Wilson Observatory over a period of a few decades, have revealed apparent variations of about 0.4 " that are correlated with the solar cycle. We note that the radius definition used for the analysis of those magnetograms automatically converts intensity variations near the limb into apparent radius variations. A change in the average temperature structure of the quiet Sun can be ruled out as the source of these variations, since such a change would need to be very significant and would lead to other easily measurable consequences that are not observed. We show that plage emission near the solar limb associated with the magnetic activity variation during a solar cycle produces apparent radius changes of the correct sign. The use of plane-parallel or spherically-symmetric models to describe the faculae gives apparent radius variations that are a factor of 4-10 too small in magnitude. If the Mt. Wilson results are correct, then this implies that the small-scale structure of faculae produces limb extensions that are considerably larger than those returned by a plane-parallel or spherically-symmetric model.
\end{abstract}

Key words. line: formation - Sun : activity - Sun: atmosphere - Sun: faculae, plage - Sun: fundamental parameters

\section{Introduction}

Solar radius measurements have a long history and the methods used are rather diverse. The accuracy of the older records is very uncertain, and the results depend significantly on the measurement procedure and the instrument used. Even measurements with the same type of instrument show a remarkably large spread, indicative of the difficulty and rather poor intrinsic precision of this type of measurements. Nevertheless, by virtue of the high number of measurements, often radius variations are claimed that are much smaller than the intrinsic measurement precision of the instrument. Overviews of the most prominent historic radius measurements and the methods applied can be found in Wittmann (1977) and Toulmonde (1997). After a careful re-analysis of a large set of historic radius measurements Toulmonde (1995), summarized in Toulmonde (1997), concludes that there is no evidence for any of the secular or long-period radius changes claimed to have been observed during the last three centuries.

Even recent measurements, frequently aiming at detecting radius variations during a solar cycle with sophisticated special-purpose instruments, have an accuracy that is only comparable to the maximum radius variations that are claimed by older measurements. Moreover, it is even unclear whether radius variations on solar-cycle time scales, if they occur at all, are correlated with the solar cycle or anti-correlated, as most measurements seem to indicate. In this paper, we comment in particular on the $0.4^{\prime \prime}$ apparent radius variation between solar activity minimum and maximum found by Ulrich \& Bertello (1995), on the basis of a 13-year sequence of Mt. Wilson magnetograms. They stress, however, that the measured variation concerns the apparent solar radius not the true radius, and that its physical cause is most likely a change in the temperature profile of the Sun's atmosphere with the solar cycle. Even though it is not possible to unambiguously define the true radius, one is inclined to associate the true radius with a mass density criterion rather than with an emergent intensity. Unfortunately, one cannot measure the density radius directly, and radius values based on intensity measurements present a poor proxy, since their variations do not necessarily imply equal variations of the density radius. Similarly, even though we use the term 'actual limb positiont', there is no such thing as the limb. It is commonly defined as the inflection point of the $I(r)$-curve, which is located close to the point where the intensity has decreased by $50 \%$ from the value just inside the limb.

Here we test how large a change in the quiet solar atmosphere outside active regions would need to be to reproduce these measurements and whether the claimed variation in the 
solar radius can be reproduced by changing the area coverage of active region plage over the solar cycle. The definition of the solar radius as employed by Ulrich \& Bertello (1995) and previously by LaBonte \& Howard (1981), who found no significant radius variation with time, is very sensitive to changes in the center-to-limb variation of the intensity, which may be caused, e.g., by the cycle-related variation of the network and plage coverage of the solar surface. Such filling factor variations imply a change in the (average) temperature profile of the Sun's atmosphere. In principle, a re-analysis of the Mt. Wilson data for active and inactive latitudes separately could confirm or reject our suggestion that the measured radius variations are due to plage filling factor variations in the course of the solar cycle. Unfortunately, the accuracy of the radius measurements would then decrease and consequently it would become prohibitively difficult to identify the cause of possible differences.

\section{Mt. Wilson data acquisition and processing}

Since the data acquisition and analysis, in particular the determination of the solar limb location, play an important role in our argumentation, we briefly describe the Mt. Wilson data acquisition and processing for the sake of clarity. The data used by Ulrich \& Bertello (1995) for the radius analysis are part of the Mt. Wilson archive of daily magnetograms taken since 1966. In order to be useful for radius measurements, the instrument system should not be changed in any way over a long period of time, which is why only the data obtained after 1981 can be used. As described by LaBonte \& Howard (1981), who as a result of several instrumental changes during the period analyzed did not find any significant radius changes, several magnetograms are obtained routinely each day by scanning the image of the Sun over a 12.5" square aperture by means of the coelostat mirrors. Each magnetogram is built up by scanning the Sun's image over the spectrograph entrance aperture in alternating directions; the direction of the scan lines (primary direction) is adjusted once a day to be perpendicular to the solar rotation axis; the variation of the direction of the rotation axis in the course of a day can be ignored. Initially the aperture is positioned well off the north or south limb (randomly chosen) and each magnetogram is built up by successive scans back and forth in the primary direction.

The data system continually samples the intensity and circular polarization in two spectral passbands, with a fixed central separation of $7.9 \mathrm{pm}$ and a width of $6.5 \mathrm{pm}$ (Howard et al. 1983); by means of servo-control these passbands are maintained to be equally illuminated on opposite wings of the FeI525.02 nm line, thereby automatically correcting for all Doppler shifts that influence the position of the line (as long as the line exhibits the same asymmetry everywhere). It takes about 55 min to obtain a single magnetogram, so that conditions may change considerably during data acquisition. Sophisticated correction procedures are applied to correct for most effects that may influence the final result (see LaBonte \& Howard 1981).

One magnetogram per day is used for the radius analysis presented by Ulrich \& Bertello (1995). For each scan line they define the limb as the location of the point that has an intensity of $25 \%$ of the disk center intensity; due to the rather wide entrance aperture this position can differ significantly from the conventional limb position defined by the inflection point of the $I(r)$-curve. In order to eliminate instrument backlash effects, the E-W scan lines and the W-E scan lines are analyzed as separate sets, and for each of them a least squares fit is made to determine the exact location of the disk center and the solar radius. Finally, the ephemeris value of the solar radius is subtracted from the measured solar radius and the resulting 13 -year data series is linearly detrended. The remaining radius variations have a peak-to-peak value of about $0.4^{\prime \prime}$ and are correlated with the solar activity cycle, the largest radius occurring near activity maximum.

\section{Computational methods and basic results}

\subsection{Models}

We employ semi-empirical models for the quiet Sun (FAL-C), magnetic network (FAL-F), and plage (FAL-P) from Fontenla et al. (1991). These semi-empirical models represent a spatial and temporal average of the radiation temperatures (not of the actual local temperatures, according to e.g. Carlsson \& Stein 2002) of plage and network regions; they are very similar to the quiet-Sun model. One might argue that models representing network or plage flux tubes, such as the ones derived by Solanki \& Brigljević (1992), deviate substantially more from the quiet Sun than FAL-F or FAL-P, so that the resulting line profiles will also deviate much more from the FAL-C results. However, that is of minor interest in the present context since the large aperture of the Mt. Wilson instrument causes significant spatial averaging anyway; as long as the product of network or plage filling factor and the corresponding intensity enhancement w.r.t. the quiet-Sun remains within reasonable observational limits it is not particularly relevant how we define the network and plage models. A complication may result from ignoring geometry effects: towards the limb an average network or plage model like FAL-F or FAL-P may look significantly different from a bunch of discrete magnetic elements embedded in and interacting with a quiet-Sun environment (cf. Solanki et al. 1998). On the whole, we expect that the main effect of taking into account flux tubes will come from their partial evacuation. Thus we expect the limb extension due to plage to be somewhat smaller than the values we obtain. The error should be on the order of $\alpha Z_{\mathrm{W}}$, where $\alpha$ is the magnetic filling factor $(\approx 0.1)$ and $Z_{\mathrm{W}}$ is the Wilson depression $(\approx 150 \mathrm{~km})$, making a probable error of $15 \mathrm{~km}$ or $0.02^{\prime \prime}$. However, this is just a rough estimate. We shall return to this point in Sect. 6.

In order to have a broader diagnostic, we also include the Fe I $1564.85 \mathrm{~nm}$ line, which is formed in the deep photosphere. We expect that this line produces much smaller apparent radius variations than Fe I $525.02 \mathrm{~nm}$ by virtue of the significantly flatter center-to-limb variation of the intensity in the infrared. Nevertheless, this particular line would not be suitable for measuring radius variations by the Mt. Wilson method. Due to its larger magnetic field sensitivity even network fields produce measurable line splitting; consequently, on the one hand this line is not suitable for making conventional magnetograms and 
on the other hand it suffers from significant field-induced line profile variations that in turn would falsify the solar radius determination. A non-magnetic infrared line comparable to Fe I $1564.85 \mathrm{~nm}$ or the infrared continuum would provide better radius measurements. A suitable collection of such lines may be found in the papers by Solanki et al. (1990) and Ramsauer et al. (1995).

The atomic model is kept as simple as possible: it contains the basic ingredients that determine the statistical equilibrium of neutral iron, which is heavily dominated by overionization as a result of the superthermal UV radiation field in most parts of the photosphere, and the behavior of the Fe I $525.02 \mathrm{~nm}$ and $1564.85 \mathrm{~nm}$ lines in particular. We stress that, although a fair reproduction of the observed quiet-Sun line profiles is a prerequisite, the differential effects that we are considering here are quite insensitive to deviations from the "exact" solar line profile.

\subsection{Radiative transfer computations}

The non-LTE radiative transfer computations are done in spherical geometry by means of the RH code (Uitenbroek 2001), which provides a natural way to compute line profiles beyond the solar limb. The computational grid consists of a set of limb rays parallel to the central ray (disk center) and tangent to the spherical shells defined by the height grid of the atmospheric model (Mihalas 1978, p. 253). These rays are necessarily all located very close to the limb, since the atmosphere models cover only the innermost $2000 \mathrm{~km}$ or so of the Sun's atmosphere. These limb rays are complemented by a number of core rays that are evenly distributed between disk center and the innermost limb ray. The non-LTE computations are performed for each model separately, which means that radiative interactions between plage or network and their quiet-Sun environment are ignored.

\subsection{Basic non-LTE properties}

Figures 1 and 2 illustrate the basic non-LTE formation properties of the Fe I $525.02 \mathrm{~nm}$ and Fe I $1564.8 \mathrm{~nm}$ lines. The population departure coefficients, defined as $\beta_{i}=n_{i} / n_{i}^{*}$, where $n_{i}$ represents the actual population of level $i$ and $n_{i}^{*}$ represents the corresponding LTE population, collectively drop below unity in the photosphere due to the superthermal ionizing UV radiation field originating in the deep photosphere. The results for model FAL-C, which are shown in Fig. 1, are representative for all our models. The diamond and the triangle indicate the respective line center optical depth unity locations: both spectral lines are formed below the depth where the departure coefficients of their upper and lower levels start to diverge, so that their source functions (Fig. 2) remain in LTE throughout their formation region. Due to the strong overionization of iron, indicated by $\beta<1$ in the photospheric layers (Fig. 1), the nonLTE formation region lies somewhat deeper than in LTE. This also implies that the computed lines are somewhat weaker in NLTE than in LTE. This effect is particularly pronounced for Fe I $525.02 \mathrm{~nm}$, the stronger of the two lines.

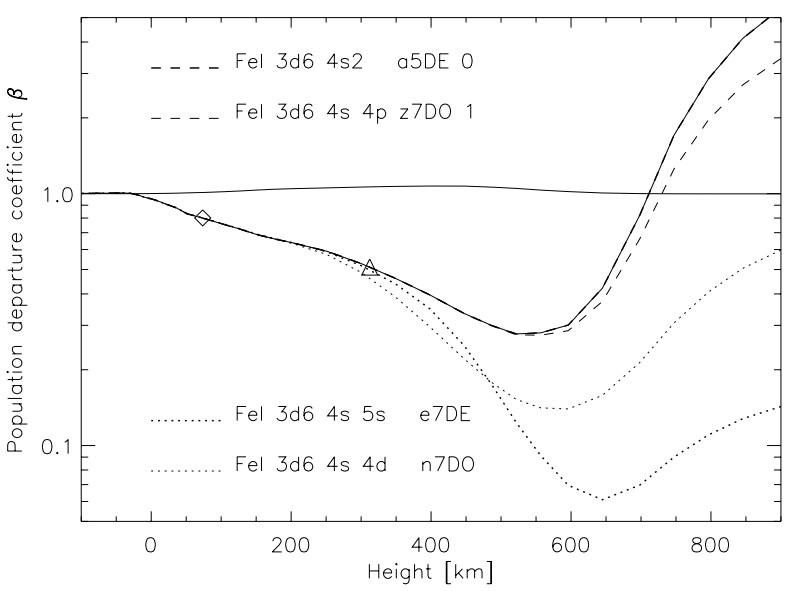

Fig. 1. Population departure coefficients, $\beta$, for the atomic energy levels relevant for the spectral lines considered; drawn here are the results for the FAL-C model, which are representative for all models. The neutral (once-ionized) iron ground level is indicated by the thick (thin) solid curve. The lower and upper levels of the $525.02 \mathrm{~nm}(1564.8 \mathrm{~nm})$ line are indicated by thick respectively thin dashed (dotted) curves and the line center optical depth unity location is indicated by a triangle (diamond). The thick dashed curve is not visible; it coincides with the thick solid curve.

Figure 3 shows the FAL-C limb profiles for both spectral lines. They are again typical for the profiles resulting from the other models as well. The figure reveals that near $500 \mathrm{~nm}$, representative of the entire visible part of the spectrum, the continuum limb lies at a height of about $400 \mathrm{~km}$ above $\tau=1$ at vertical incidence (which is usually taken to define $z=0$ in atmospheric models), whereas in the $1.6 \mu$ infrared it lies near $300 \mathrm{~km}$. At the centers of the two lines the limb lies near 650 , respectively $450 \mathrm{~km}$.

\subsection{Comparison of computed and observed line profiles}

The computations presented here employ the 3 times enhanced oscillator strength of Fe I $525.02 \mathrm{~nm}$ that was also used and explained by Bruls \& von der Lühe (2001). This measure is necessary to reasonably reproduce the observed line profile in our non-LTE computations. As a consequence, the corresponding LTE line profile would become much stronger than observed. Even though we use this enhancement over the published experimental value, which is corroborated by means of LTE line profile fits to the observed solar spectrum, we do not want to criticize the experimental value at all. More likely, the onecomponent, necessarily static, plane-parallel (homogeneous) semi-empirical atmospheric models are inadequate; most are the result of LTE and even non-LTE analyses of a variety of diagnostics, but always based on spatially and temporally averaged observations. As has been shown repeatedly, the spatial and temporal average of the spectrum resulting from a dynamic 2-D or 3-D structured atmosphere is not the same as the spectrum resulting from the spatially and temporally averaged version of that atmosphere, so that it should not come as a surprise 


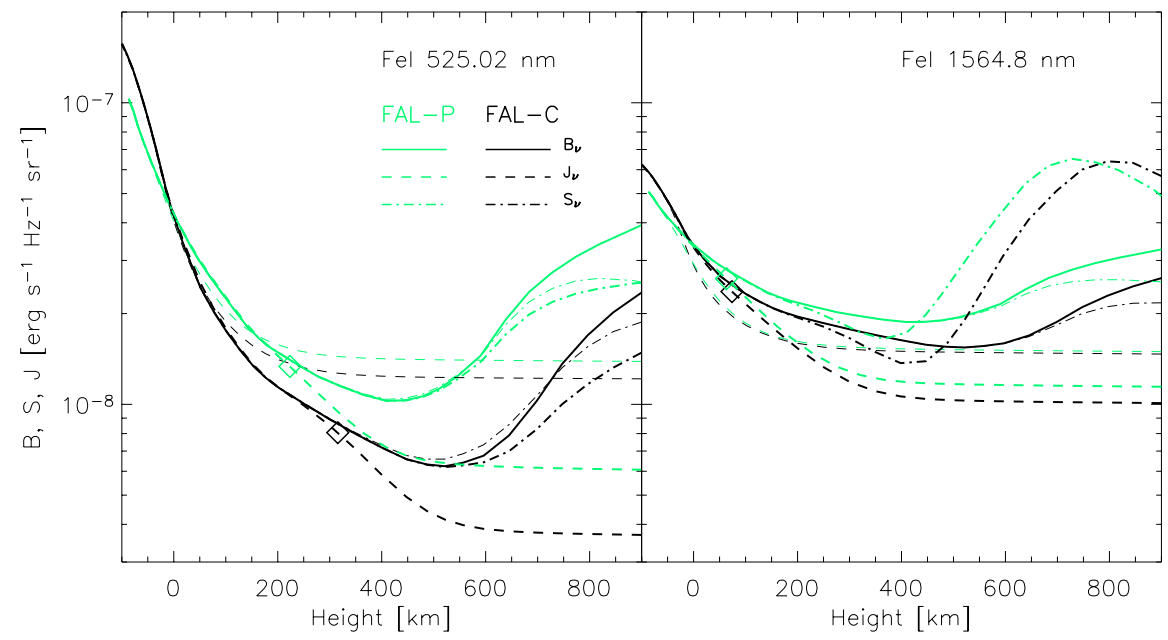

Fig. 2. Basic formation properties of Fe I $525.02 \mathrm{~nm}$ (left) and Fe I $1564.8 \mathrm{~nm}$ (right), represented by source function $\left(S_{v}\right.$, dot-dashed) and angle-averaged intensity $\left(J_{v}\right.$, dashed), relative to the Planck function $\left(B_{v}\right.$, solid curves) for models FAL-C (black), and FAL-P (gray), both at the line center wavelength (thick curves) and in the nearby continuum (thin curves). The diamonds on the $J_{v}$-curves mark the optical depth unity points at line center. The curves for the FAL-F model are not drawn here for clarity, since they generally run very close to the FAL-C curves.
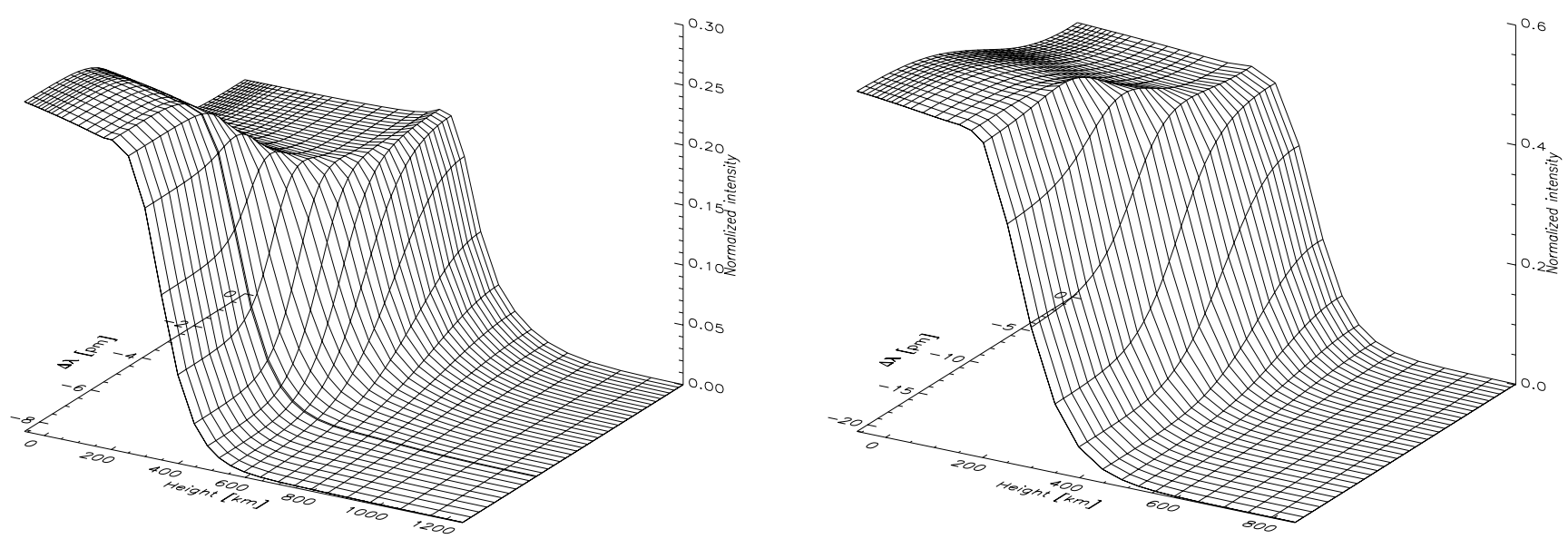

Fig. 3. Limb profiles - intensities as a function of height and wavelength - from the FAL-C model for Fe I $525.02 \mathrm{~nm}$ (left) and Fe I $1564.8 \mathrm{~nm}$ (right). The wavelength is measured in pm from line center and runs from the continuum to line center $(\Delta \lambda=0)$, and the height is measured in $\mathrm{km}$ above standard $(500 \mathrm{~nm})$ optical depth unity. The intensities are normalized to the respective disk-center continuum intensities. The profiles for the other models differ only quantitatively from the FAL-C results.

that a non-LTE computation for such an average atmosphere results in poor line profiles. The factor of 3 enhancement roughly counteracts the non-LTE population depression as shown in Fig. 1, in essence mimicking LTE populations again. For the infrared line, which is formed somewhat deeper, in a region where the underpopulation due to photoionization is still moderate, this discrepancy is negligible.

\subsection{Disk-center contrast}

From Fig. 4, which shows the wavelength-dependent intensity contrast due to FAL-F or FAL-P relative to FAL-C, it becomes clear that the disk-center intensities of FAL-C and FAL-F are very similar, but not exactly equal. In the visible continuum FAL-F is slightly less bright than FAL-C (within 1\%), while in the line wings it crosses over to higher intensities, reaching values of up to $18 \%$ near line center. A FAL-F component is therefore virtually invisible against the quiet-Sun disk-center background, except in the line core. On the other hand, FAL-P is about $4 \%$ brighter in the continuum, up to $75 \%$ in the steep parts of the line wings and slightly less at line center, so that it manifests itself at all wavelengths.

In going towards the limb the largest changes occur in the continuum: the FAL-F and FAL-P intensities are significantly larger than in FAL-C. The line itself becomes slightly brighter in FAL-F, whereas in FAL-P it becomes somewhat less bright relative to FAL-C.

In the infrared (right-hand panel of Fig. 4) FAL-F is rather inconspicuous (relative to FAL-C) over the entire disk in the continuum as well as in the line, whereas FAL-P has enhanced line intensities everywhere and enhanced continuum intensities only near the limb. However, as expected, the intensity enhancements are nearly an order of magnitude smaller than in 


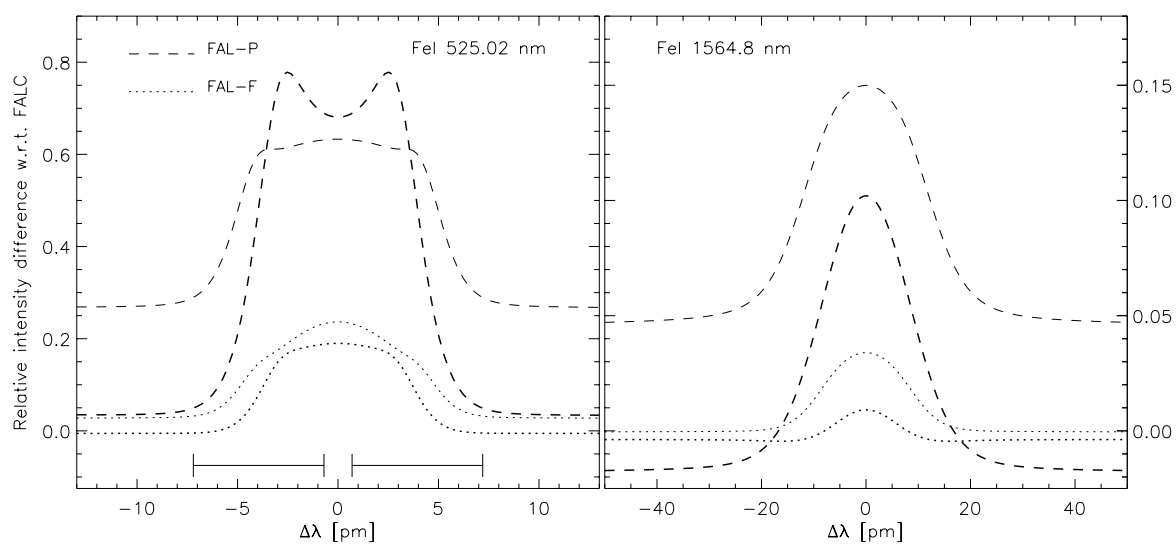

Fig. 4. Contrast of models FAL-P and FAL-F relative to FAL-C across the entire line profile at disk center (thick curves) and at the limb (thin curves). Near the limb the contrast is insensitive to the exact position, so that the curves plotted are equally valid for zero height, continuum limb height and line center limb height. The two regions delineated by the vertical lines mark the Mt. Wilson bandpass; it essentially covers the entire $525.02 \mathrm{~nm}$ line.

the visible (note the different abscissa scales of the two panels).

\section{Post-processing of the computed intensities}

In order to test our suggestion that the measured radius variations may be due to intensity enhancements related to plage regions we convert the synthetic line profiles to limb extensions by applying the Mt. Wilson observation and data analysis procedures. For each model separately the intensities have to be integrated over the appropriate wavelength interval and convolved with the 12.5 arcsec square aperture; the limb position is then defined by the $25 \%$ intensity level (relative to disk center). For the spectral passband we use a simple boxcar profile with the appropriate width, but we verified that other profiles, in particular Gaussian ones, result in nearly identical limb positions.

The choice of whether to use the FAL-F or FAL-P diskcenter intensity or just the FAL-C disk-center value when comparing the facular models at the limb depends on the aim of the computations. If we aim to test how large a limb extension is produced by a change in the average stratification of the Sun's atmosphere as a whole we need to compare like with like (e.g., FAL-F everywhere). In order to appreciate the influence of the network and plage we need to compare with FAL-C at disk center. Here we use these two extremes side by side. In reality, however, the disk-center intensity also incorporates a contribution from the magnetic network. This network component is slightly brighter than the non-magnetic quiet Sun, but due to its small filling factor, adding a network component to the quiet Sun would result in a negligible increase of the disk-center intensity. We note that by ignoring the network contribution we slightly overestimate the true limb extension values.

Apart from the choice of the disk-center intensity used for the normalization, there are two purely observational issues that need to be discussed since they affect the radius measurements.

- The position angle dependence of the radius. The orientation of the square aperture, which is scanned over the entire solar disk in about 150 lines, is kept aligned with the solar rotation axis. Therefore how the solar limb cuts through the aperture varies with its position along the solar limb; the

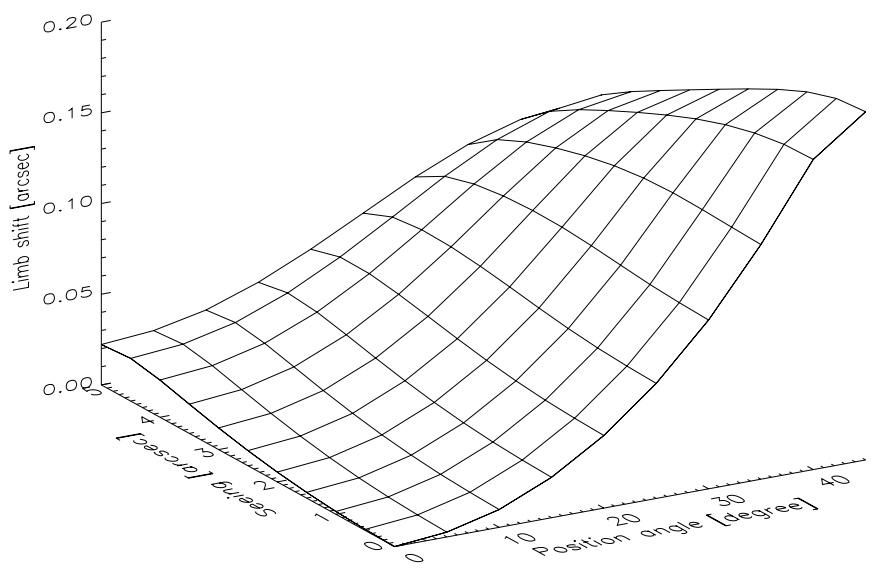

Fig. 5. Limb shifts, in arcseconds, as a function of position angle, $\phi$, of the aperture and as a function of the FWHM of the seeing disk. The position angle only runs from $\phi=0^{\circ}$ to $45^{\circ}$, since the behavior is symmetric around $\phi=45^{\circ}$. The limb position at $\phi=0^{\circ}$ observed at ideal seeing serves as the reference.

resulting position-angle dependence of the radius measurements is discussed in Sect. 4.1.

- Seeing. Since the aperture of the Mt. Wilson instrument is large compared with the typical seeing disk size, we expect that the influence of seeing will be very moderate. In Sect. 4.2 we explore the role of seeing by means of a simple model.

Since the Mt. Wilson radius measurements are affected by both effects at the same time and since the effects are not completely independent we choose to consider their joint effect instead of studying them separately in idealized experiments.

\subsection{Position-angle dependence}

At latitudes of $0^{\circ}$ and $90^{\circ}$, resp. at the equator and at the poles, the aperture of the Mt. Wilson instrument is aligned with the limb; at all other latitudes the limb makes an angle of up to $45^{\circ}$ with the sides of the aperture. Therefore, the intensity $I_{\mathrm{A}}$ integrated over the aperture depends on the radial position $r$ of the center of the aperture as well as on the position angle $\phi$ along the solar limb. 
The resulting position-angle dependence of the radius measurements has mirror-symmetry about the position angle of $45^{\circ}$. This position-angle dependence is solely a measurement effect that has nothing to with the actual difference between the polar and equatorial radius, which is generally accepted to be smaller than about $8 \mathrm{~km}$, with an average observational value of about $6 \mathrm{~km}$ (Godier \& Rozelot 2000).

To estimate to what extent the Mt. Wilson radius measurements are affected by the fact that the aperture is always oriented parallel to the solar rotation axis we apply the Mt. Wilson data acquisition and analysis procedures to our synthetic FAL-C intensities. Both the integrations over the spectral passband and over the aperture are performed numerically to obtain $I_{\mathrm{A}}(r, \phi)$ and from that we determine the limb position as a function of $\phi$. In addition, seeing influences the measurements; we model this in Sect. 4.2, resulting in a plot of the shift of the limb position as a function of position angle $\phi=0$ and seeing parameter relative to the case of ideal seeing and $\phi=0$ (Fig. 5). First consider only the curve for ideal seeing (seeing parameter zero). We see that the radius measured at a position angle of $45^{\circ}$ is about $0.2 \operatorname{arcsec}$ larger than at $\phi=0^{\circ}$. The difference between the observational solar radius - which is an average over all position angles - and the ideal radius determined with the aperture always aligned with the solar limb, should be significantly smaller than these $0.2 \mathrm{arcsec}$. It is therefore safe to ignore this dependence in the following analysis. In addition, the average latitude of active-region faculae is probably close to $20^{\circ}$, where the influence of the position angle effect is negligible anyway. Latitudinal migration of faculae (butterfly diagram) over the solar cycle is also neglected.

\subsection{Seeing}

Seeing is in general a combination of, on the one hand, image motion and distortion and, on the other hand, intensity modulation. At the spatial scales relevant to this analysis, however, it is essentially limited to image motion, which at the solar limb results in a rapid variation of the portion of the $12.5^{\prime \prime}$ by $12.5^{\prime \prime}$ aperture that is covered by the solar disk, hence in a variation of the detector signal. The measurements at Mt. Wilson are made by means of photomultiplier tubes and the spatial scanning speed of $78^{\prime \prime}$ per second results in an effective exposure time for each pointing direction - equivalent to location on or near the solar disk only if there were no seeing-induced image motions - of $127 \mathrm{~ms}$ (Howard et al. 1983). This exposure time is significantly longer than typical exposure times required to freeze seeing-induced motions, so that intensity variations due to image motion largely cancel out in the temporal average. The influence on the radius measurements would be exactly zero if the intensity drop near the limb were a perfect step function, but the slight intensity gradient just inside the limb on average causes a minor apparent radius increase due to incomplete cancellation.

We model the influence of seeing on the observed intensities by means of an additional spatial smearing with a Gaussian profile with a FWHM that defines the seeing disk. From those intensities we derive limb shifts w.r.t. the case with ideal seeing. Figure 5 displays these limb shifts as a function of position angle along the solar limb and as a function of the FWHM of the seeing disk. It demonstrates that seeing works differently for different position angles: near $\phi=0^{\circ}$ it increases the radius, whereas near $\phi=45^{\circ}$ it actually reduces the radius. The Mt. Wilson magnetograms represent an average over all position angles and should be relatively insensitive to seeing variations. We conclude that for a moderate average seeing of $2^{\prime \prime}$ the limb will be shifted outward by about 0.1 arcsec on average, and that the average apparent radius increases only very slowly with the seeing parameter, since the effects at different position angles largely cancel out. Considering that the acquisition of a complete Mt. Wilson magnetogram takes about 55 min we may expect the average seeing conditions at Mt. Wilson to be imprinted on most magnetograms: exceptional seeing for such an extended period of time, leading to a slightly smaller measured solar radius, can almost certainly be excluded, and magnetograms obtained at significantly poorer seeing may have been omitted from the data set from the start or discarded afterwards. Seeing causes the absolute radii derived from the observations to be too large by roughly $0.1 \mathrm{arcsec}$, but the long effective exposure time of 55 min significantly smooths out seeing variations over a single image and from image to image, so that the seeing-induced variation of the measured solar radii should be smaller than the absolute error introduced by seeing. Seasonal and daily variation of average seeing conditions may be responsible for part of the remaining scatter in the radius measurements presented by Ulrich \& Bertello (1995), but for our experiments seeing is not relevant.

\section{Results}

\subsection{Synthetic limb extension observations as a function of wavelength}

Figure 6 shows the radius extensions for the entire wavelength range for both Fe I 525.02 and $1564.8 \mathrm{~nm}$, with infinite spectral resolution (solid curves) as well as for a given spectral bandpass (boxcar transmission profile). The limb extension values for $525.02 \mathrm{~nm}$ are about $640 \mathrm{~km}$ (FAL-F) and $800 \mathrm{~km}$ (FAL-P) if we assume that the disk-center intensity is also given by FAL-F or FAL-P, respectively. Thus even a significant change in the average atmosphere, as represented by FAL-F and FAL-P gives limb extensions that are only a factor of 2-3 larger than observed. Assuming network and faculae to be the main cause of the observed limb extension a more realistic definition builds on using the quiet-Sun disk-center intensity to define the limb; the corresponding limb extension values are then $860 \mathrm{~km}$ for FAL-F and $1800 \mathrm{~km}$ for FAL-P.

In order to obtain the observed apparent limb extension of $0.4^{\prime \prime}$, corresponding to about $290 \mathrm{~km}$, we would need roughly $35 \%$ or $16 \%$ excess coverage by network/faculae during activity maximum compared with activity minimum for FAL-F or FAL-P, respectively. Furthermore, since active region plage occur almost exclusively at lower latitudes, the actual coverage in the active latitude range should be even larger to compensate for the absence of plage at higher latitudes. These numbers can be compared with filling factor enhancements of 

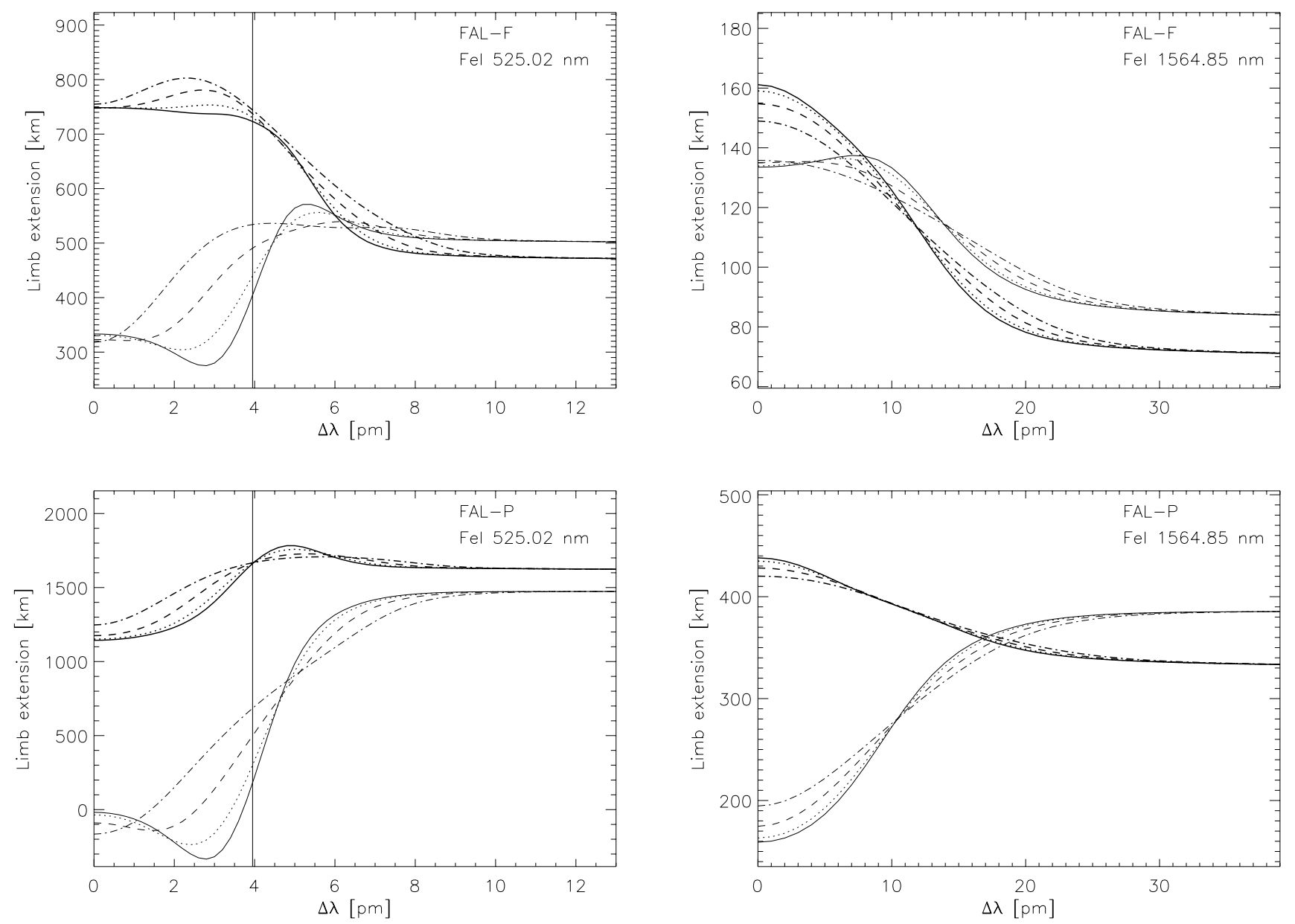

Fig. 6. Limb extensions for Fe I $525.02 \mathrm{~nm}$ (left) and $1564.8 \mathrm{~nm}$ (right) from models FAL-F (top) and FAL-P (bottom), measured relative to FAL-C. The thin curves are based on an intensity normalisation with the model's own disk-center intensity; thick curves are based on a normalisation with the FAL-C disk-center intensity. Note the large range and the occurrence of negative values in the line core for FAL-P. Solid curves correspond to the unfiltered data, the other curves result after applying a boxcar spectral transmission window with a width of 2 pm (dotted), $4 \mathrm{pm}$ (dashed) and $6.5 \mathrm{pm}$ (dot-dashed) for $525.02 \mathrm{~nm}$, resp. 6, 12 and $19.5 \mathrm{pm}$ for $1564.8 \mathrm{~nm}$. The largest filter width used for the $525.02 \mathrm{~nm}$ line corresponds to the spectral passband of the Mt. Wilson instrument, whose center position is marked by the vertical lines.

about $4 \%$ found by Solanki \& Unruh (1998) from models that reproduce the difference in total and spectral irradiance variations between solar activity maximum and minimum.

Even if we argue that due to geometry effects near the limb the apparent plage coverage fraction is larger than the actual value, it is difficult to reconcile our plage coverage fraction required near the limb with the global coverage of about $4 \%$ (based on FAL-F) found by Solanki \& Unruh (1998). When viewed away from disk center, flux tubes appear as elongated features, with correspondingly larger apparent surface coverage fraction. Additionally, we may have more than one flux tube along the line of sight, so that we have to consider the interaction with the environment and with other tubes. Therefore, a quantitative and even a meaningful qualitative demonstration of the apparent coverage fraction enhancement towards the limb is quite involved.

Obviously neither a reasonable global change in the atmospheric stratification nor the change in filling factor of network or faculae appear to be able to reproduce the observed limb extensions quantitatively.

\subsection{Limb extension as a function of aperture size}

As a demonstration of the extreme sensitivity of the radius measurements to the aperture size, we plot in Fig. 7 the synthetic limb extension measurements (at infinite spectral resolution) for a range of aperture sizes up to $20^{\prime \prime}$. The dotted vertical lines indicate the center positions of the two spectral windows of the Mt. Wilson instrument in the steepest part of the line wings; the limb extension given for that point is a rough approximation to the results obtained for the spectral window used by the Mt. Wilson instrumentation. It is immediately clear that the measurement procedure itself, which mixes real radius variations with intensity variations, is an important potential cause of the observed radius variations. Indeed, one can directly see that an increase by a factor of $1+\epsilon$ of the intensity immediately 

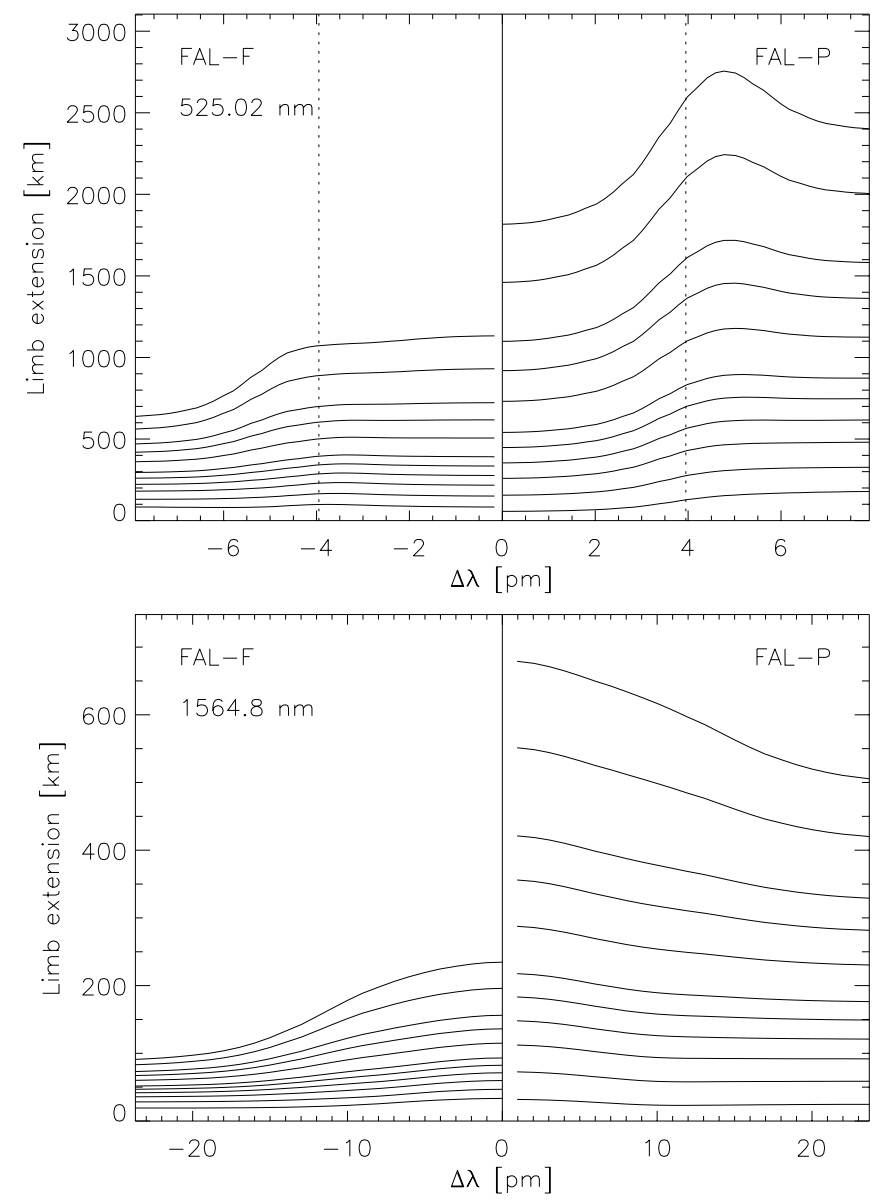

Fig. 7. Synthetic limb extension measurements as a function of wavelength (infinite spectral resolution) for Fe I525.02 nm (top) and $1564.8 \mathrm{~nm}$ (bottom) obtained for models FAL-F (left half of figure) and FAL-P (right) relative to FAL-C for square apertures of 1, 2, 3, $4,5,6,8,10,12,16$ and 20" (from bottom to top in each panel). All are based on the limb definition with quiet-Sun disk-center intensity normalization. The dotted vertical lines mark the center wavelengths of the Mt. Wilson spectral windows, roughly at the steepest wing part of the quiet-Sun line profile. The case of infinite spatial resolution is not explicitly plotted, since by virtue of the height scale definitions of the various atmospheric models this is forced to approach zero.

inside the limb is equivalent to a shift of the limb position by a fraction $\epsilon$ of the aperture size. This means that for an aperture of $12.5^{\prime \prime}$ the line wing intensity near the limb need only increase by 3 percent to cause the observed apparent radius variation.

\section{Discussion}

We have shown that the apparent radius variations observed by Ulrich \& Bertello (1995) can be fully explained by a 3\% change of the line wing intensities near the limb during the course of a solar cycle. Non-LTE radiative transfer computations for models of quiet-Sun and plage regions show that such an increase can be obtained by assuming an apparent plage surface coverage fraction of about $15-35 \%$ near the limb. This number is very high compared with realistic disk-center plage coverage fractions of a few percent. For instance, Solanki \& Unruh (1998) found that the difference between activity minimum and activity can be attributed to a $4 \%$ change in the surface coverage fraction of model FAL-F. Hence with synthetic 1-D models alone we cannot fully explain the observed apparent radius variations.

However, various sources of uncertainty still remain in the computations. One of the more intricate ones is the fact that in the radiative transfer computations in spherical symmetry the zero point of each model's height scale, by definition the height where the standard optical depth reaches unity, is located at exactly the solar radius. Pressure differences among the different models, symptomatic of a height scale mismatch, are thus automatically ignored. At photospheric heights the pressure differences between models FAL-C, FAL-F and FAL-P are equivalent to height scale offsets of up to a moderate $20 \mathrm{~km}$.

In principle, it would be easy to correct for that difference by shifting the models accordingly, were it not for the uncertain magnetic pressure that plays an important role in this balance. On the other hand, it is not quite consistent to add a magnetic field and its associated magnetic pressure to the FAL-P or FAL-F models, since these models merely represent a poorlydefined temporal and spatial average of a plage region, consisting of largely field-free material surrounding one or more (subresolution) magnetic elements. Typical values for the Wilson depression in flux tubes are of the order of a few hundred kilometers; for models like FAL-F and FAL-P this value should be reduced by the magnetic filling factor, hence it becomes substantially smaller.

One major simplification of the present modeling is the neglect of the magnetic field. It influences the results in two ways. Firstly, it changes the line profiles, which can influence the deduced limb extension. In addition, it enters into the force balance and lowers the $\tau=1$ level within the magnetic elements. To first order we expect this to be offset by a minute increase of the height at which the solar surface is located outside these elements. Roughly, this increase corresponds to $\alpha Z_{\mathrm{W}}$, where $\alpha<1$ is the magnetic filling factor and $Z_{\mathrm{W}}$ is the Wilson depression, or the amount by which $\tau=1$ is lowered in the magnetic elements. For $Z_{\mathrm{W}}=200 \mathrm{~km}$ and $\alpha=0.1$, this would give a $20 \mathrm{~km}$ higher $\tau=1$ level. A part of the effect is also due to the different heat flux and hence temperature stratification in magnetic elements and the field-free gas (Spruit 1982). However, even for an unrealistically active Sun this will only give radius changes that are far below a pressure scale height.

If we consider the Mt. Wilson results to be correct, then additional sources of limb extensions in plage are needed. These may be related to the small-scale magnetic structure of plage, but we expect mainly to its dynamics (e.g., ejection of material to higher layers within magnetic elements, Steiner et al. 1998). Neither the structure nor the dynamics can be adequately represented by the simple spherically-symmetric models that we have considered here.

Acknowledgements. We thank an anonymous referee for careful and detailed comments that improved our paper. The non-LTE radiative transfer was carried out by means of the flexible RH code written by H. Uitenbroek. A. Wittmann provided the necessary background information on radius measurements. This research has made use of 
NASA's Astrophysics Data System Abstract Service and the NIST atomic database.

\section{References}

Bruls, J. H. M. J., \& von der Lühe, O. 2001, A\&A, 366, 281

Carlsson, M., \& Stein, R. F. 2002, ApJ, 572, 626

Fontenla, J. M., Avrett, E. H., \& Loeser, R. 1991, ApJ, 377, 712

Godier, S., \& Rozelot, J.-P. 2000, A\&A, 355, 365

Howard, R., Boyden, J. E., Bruning, D. H., et al. 1983, Sol. Phys., 87, 195

LaBonte, B. J., \& Howard, R. 1981, Science, 214, 907

Mihalas, D. 1978, Stellar Atmospheres, 2nd edition (San Francisco: Freeman and Company)
Ramsauer, J., Solanki, S. K., \& Biémont, E. 1995, A\&AS, 113, 71

Solanki, S. K., Biémont, E., \& Mürset, U. 1990, A\&AS, 83, 307

Solanki, S. K., \& Brigljević, V. 1992, A\&A, 262, L29

Solanki, S. K., Steiner, O., Bünte, M., Murphy, G., \& Ploner, S. R. O. 1998, A\&A, 333, 721

Solanki, S. K., \& Unruh, Y. C. 1998, A\&A, 329, 747

Spruit, H. C. 1982, A\&A, 108, 348

Steiner, O., Grossmann-Doerth, U., Knölker, M., \& Schüssler, M. 1998, ApJ, 495, 468

Toulmonde, M. 1995, Ph. D. Thesis, Observatoire de Paris

Toulmonde, M. 1997, A\&A, 325, 1174

Uitenbroek, H. 2001, ApJ, 557, 389

Ulrich, R. K., \& Bertello, L. 1995, Nature, 377, 214

Wittmann, A. 1977, A\&A, 61, 225 